\title{
School Shootings: International Research, Case Studies, and Concepts for Prevention
}

\author{
Nils Bockler, Thorsten Seeger, Peter Sitzer, Wilhelm Heitmyer, Editors, Springer \\ Science + Business Media New York, 2013. 543 Pages. \$144.01
}

\author{
Caitlin R. Green ${ }^{1}$
}

Published online: 22 April 2017

(C) Springer International Publishing 2017

School Shootings is a comprehensive text which provides an overview of all topics relevant to a wide range of violent acts perpetrated in various educational settings Internationally. Volume editors Nils Bockler, Thorsten Seeger, Peter Sitzer, and Wilhelm Heitmyer initially developed the idea for this project during the course of their own research in Germany, which also inspired the unique international perspective this text offers. Each chapter is authored by experts from myriad professional disciplines, including law enforcement, education, counseling, research, sociology, anthropology, and psychology, and many individuals who are experienced in multiple disciplines. These countless frames of reference and extensive knowledge available in one text to examine a serious, provocative, and global topic make School Shootings a distinctive book.

The authors provide an overview of the history of violence on school grounds, including statistics and changes in terminology throughout events and generations. Multiple theoretical frameworks for what motivates violence in schools are discussed, including a sophisticated combination of social disintegration theory, youth theory, and control theory. The role of violent fantasy as both an escape from reality and a means of romanticizing the control and mastery that will be gained from perpetrating a violent act are discussed in detail.

Though the title of the book appears to indicate a focus on firearm violence, the authors include discussions of incidents

Caitlin R. Green

caitlin.r.green@gmail.com

1 Alliant International University, 10455 Pomerado Rd, San Diego, CA 92123, USA such as a case in South Africa in which a boy used a samurai sword to harm fellow students and staff members. Case studies such as this are used throughout the text to illustrate the nuances of the theories discussed in part I of the text, in relation to why some children and adolescents engage in these violent acts. Further, some cases are used in later chapters on the role of media and prevention strategies as references. These case studies are important in creating an understanding of both the origins and the conceptual structures of the theories presented.

Of the many unique components School Shootings offers the reader, its focus on media-related issues stands out. Mediatization is a term used in the chapters on media reporting, to reflect the manner in which media sources alter or emphasize different aspects of a story to sensationalize it. These chapters discuss the effects of media portrayal (such as the "Columbine Effect") on society, and on potential future school shooting perpetrators. In addition, the effects of violent media content (e.g., violent video games, films, and television shows) on potential perpetrators of shootings is examined, given the pop culture association between viewing violent content and violent behavior.

The final chapters are devoted to prevention and response strategies for school violence. Many of the suggested approaches include identifying threats and at-risk students early, taking threats seriously, and addressing incidents of bullying in schools immediately. School Shootings is a comprehensive and thoughtful text which answers a multitude of questions raised by the cultural phenomenon of school related violence. It has utility for practitioners, law enforcement, schools, and risk/threat assessment specialists, as well as parents or other concerned laypeople who would like to know more about the topic. Though it is written in a highly scientific, textbook format in some chapters, others are highly accessible to any reader and provide a diverse, global perspective. 